Infect Dis Clin North Am. 2017 September ; 31(3): 551-559. doi:10.1016/j.idc.2017.05.007.

\title{
Prevention of Central Line-Associated Bloodstream Infections
}

\author{
Taison Bell and Naomi O'Grady \\ Critical Care Medicine Department, National Institutes of Health
}

\begin{abstract}
Central venous catheters (CVC) are commonly used in critically ill patients and offer several advantages to peripheral intravenous access. However, indwelling CVCs have the potential to lead to blood stream infections, with the risk increasing with an array of characteristics such as catheter choice, catheter location, insertion technique and catheter maintenance. Evidence-based guidelines have led to a significant reduction in the incidence of blood stream infections associated with CVCs. The combination of guideline implementation combined with newer technologies has the potential to further reduce morbidity and mortality from infections related to CVCs.
\end{abstract}

\section{Keywords}

CLABSI; CRBSI; Central Line; Central Venous Catheter; Blood Stream Infection

\section{Introduction}

Central venous catheters (CVC) are often essential in the care of the critically ill patient.

They allow safe administration of intravenous medications that cannot be given peripherally, aid in the administration of intravenous fluid resuscitation, and help in monitoring hemodynamic parameters in the management of patients with syndromes such as septic shock, cardiogenic shock, decompensated heart failure and pulmonary hypertension. Despite the benefits of CVCs, they also serve as potential portals for localized and systemic bloodstream infections. For this reason, considerable effort has gone into reducing the incidence of bloodstream infections from CVCs.

\section{Definitions}

There are two major definitions used to describe bloodstream infections related to CVCs: catheter-related bloodstream infection (CRBSI) and central line-associated bloodstream infection (CLABSI). CRBSI is a clinical definition based on clinical criteria related to a specific patient in which the diagnosis is being considered. This definition is more often used for research, and in some cases of clinical care, since it requires specialized

Corresponding Author: Taison Bell: taison.bell@ nih.gov.

Publisher's Disclaimer: This is a PDF file of an unedited manuscript that has been accepted for publication. As a service to our customers we are providing this early version of the manuscript. The manuscript will undergo copyediting, typesetting, and review of the resulting proof before it is published in its final citable form. Please note that during the production process errors may be discovered which could affect the content, and all legal disclaimers that apply to the journal pertain.

Disclosures: The authors do not report conflicts of interest relevant to the preparation of this text. 
microbiological techniques to specifically identify the catheter as the source of bacteremia that may not be available in all hospitals. In contrast, the diagnosis of CLABSI is a simplified definition based on surveillance criteria that identify bloodstream infections in patients with CVCs in which there is no other obvious secondary source for bacteremia. [1] [2]. The CLABSI definition has the potential to overestimate the true incidence of CRBSI, since many primary bloodstream infections do not have an obvious secondary source. However, in the years since the CDC instituted mucosal barrier injury as a category for secondary sources of bacteremia this overestimation has been reduced. In addition, since many states now require public reporting of hospital CLABSI rates and the Centers for Medicare and Medicaid Services instituted financial penalties for hospital reimbursements for CLABSI, there is more granularity in the reviews of bloodstream infections in some institutions, and efforts are now made to thoroughly investigate the possibility of secondary sources that, in years past, may not have occurred. These public policy changes and financial incentives to produce low CLABSI rates have raised concerns that partially subjective surveillance definitions (e.g. the NHSN definition) applied inconsistently could be exploited or be prone to subconscious cognitive bias to lower infection rates [3]. Since CLABSI is the more commonly used definition for quality initiatives, it will be the focus of this chapter. However, it is important to understand the differences between the two definitions (Table 1).

CLABSIs are an important cause of morbidity and mortality in the intensive care unit, and lead to increased costs to the healthcare system. Although there has been a $46 \%$ reduction in CLABSI rates in the United States between 2008 and 2013, an estimates 30,100 CLABSIs still occur in intensive care units and acute care wards each year[4]. Other studies have estimated CLABSIs account for a range between 84,000 - 204,000 infections per year, resulting in up to 25,000 preventable deaths at a cost of up to 21 billion dollars per year[5]. There are several measures one can take to decrease the incidence of CLABSI, and the introduction of the first widely adopted set of guidelines for the prevention of CLABSI in 2002 has led to a substantial reduction in the incidence of CLABSI [6]. Between 2001 and 2009 the incidence of CLABSI declined by $58 \%$ with a reduction in Staphylococcus aureus infections of 73\%[7]. The updated guideline released in 2011 [1] along with newer studies highlight further new strategies to reduce the cost and burden of CLABSI even further.

\section{Closed ICU vs Open ICU}

Intensive care is frequently practiced in multidisciplinary fashion, where the primary treatment team is tasked with providing care for patients with the input from specialty providers, pharmacists, therapists, nutritionists and other healthcare professionals. Such an environment can lead to differing opinions on the approach to care and methods to implement plans of care. In an effort to centralize critical decision making for critically ill patients and standardize care, many intensive care units have implemented a "closed unit" model in which the intensive care unit team assumes primary responsibility for the patient to implement diagnostic and treatment decisions. The benefits of this model include streamlining treatment decisions and an enhanced ability to implement treatment protocols. This approach may also lead to better clinical outcomes for hospital-acquired conditions. A single center study demonstrated that implementing a closed-unit model led to a $52 \%$ 
reduction in ventilator associated pneumonia $(\mathrm{CI}=49.1-54.9 \%)$ and a $25 \%$ reduction in CLABSI ( $\mathrm{CI}=22.5-27.5 \%)$ - though the latter finding was non-significant [8].

\section{Site selection for catheterization}

There are three commonly used sites for central venous catheterization: the internal jugular vein, the subclavian vein, and the femoral vein. Each are associated with infectious, thrombotic and mechanical complications, with each risk differing according to site of insertion [9]. The subclavian site is the preferred site for the sole purposes of reducing CLABSI [10] However, there are many other practical considerations when determining the site of catheter placement. Patients may not be suitable candidates for subclavian access if they have coagulopathies or anatomical considerations such as lymphadenopathy distorting normal anatomical features. In acute situations where vascular access is needed to be rapidly obtained, femoral catheterization may be preferred to avoid the risk of pneumothorax with either subclavian or internal jugular catheterization. Lastly, for patients with end stage renal disease — or those at risk for its development—subclavian catheterization should be avoided given the risk of subsequent subclavian stenosis complicating long term arteriovenous fistula access[1].

Femoral catheters should be avoided, when possible, because of their higher rates of infectious and thrombotic complications compared to the internal jugular and subclavian sites $[1,10,11]$. Femoral catheters are also associated with a higher rate of deep venous thrombosis compared to subclavian and internal jugular access[10, 12]. Although there may be good reasons to select the femoral site for insertion of a CVC in the acute setting, it may be prudent to select an alternative site if the catheter is anticipated to stay in place for more than 2 days.

\section{Do all catheter placements require ultrasound guidance?}

The use of real-time two-dimensional ultrasound for the placement of CVCs substantially decreased mechanical complications and reduced the number of attempts required for successful cannulation and failed attempts at cannulation compared with the standard landmark placement $[13,14]$. Patients that have catheters placed after several failed attempts are more likely to develop complications related to their catheter than patients whose initial attempt was successful. For this reason, all efforts should be made to insure successful cannulation and bedside ultrasound should be used.

\section{What kind of venous catheter should be used?}

The type of venous catheter used can have an influence on the likelihood of bacterial colonization and subsequent infection. Two major decisions on the type of catheter to use include single-lumen versus multi-lumen catheters and the use of catheters impregnated with antibiotics or antiseptics.

Single versus multi-lumen catheters-Limited data exist regarding the risk of CLABSI with singe versus multi-lumen catheters. While multi122 lumen catheters are convenient for patients requiring multiple infusions or blood draws for laboratories, they also provide additional potential pathways for infection. Multiple studies have shown 
increased infections in multi-lumen catheters compared to single-lumen catheters in varied patient populations, including the critically ill and cancer patients[15-17]; however a metaanalysis did not detect a significant difference when excluding many studies assessed as lowquality[18]. On balance, guidelines recommend using a catheter "with the minimum number of ports or lumens essential for the management of the patient"[1]. This is a reasonable approach rather than abandoning the convenience of multi-lumen catheters.

Antibiotic and Anti-Infective-Impregnated Catheters-Catheters impregnated with antiseptics (chlorhexidine/silver sulfadiazine) or antibiotics (minocycline/rifampin) have been shown to reduce the risk of CLABSI and potentially decrease hospital costs associated with CLABSI despite the additional cost of acquiring the more expensive catheters [19-21]. A recent meta-analysis demonstrated a $2 \%$ absolute risk reduction and relative risk of 0.62 $[22,23]$. The benefits of using impregnated catheters varies according to clinical setting, with the most significant benefits being realized in settings that are higher risk for CLABSI (e.g. ICU) than in settings in which the risk for CLABSI is low (e.g. general ward). For this reason, and in combination with the additional cost these catheters add, widespread use across all clinical settings has not been broadly recommended. However, in targeted settings such as the intensive care unit and in areas in which CLABSI rates remain higher than institutional goals, antibiotic or antiseptic impregnated catheters are recommended for routine use. [1].

Catheter Removal-The risk of central venous catheter colonization increases with catheter duration, and catheter colonization is the precursor to CLABSI. Thus, one of the most effective ways to reduce CLABSIs is to remove central venous catheters as soon as they are no longer necessary for the care of the patient. Observational studies suggest that a systemic approach to addressing the need for continued catheter use on a daily basis is highly effective[24, 25]. Such approaches include incorporation on a daily goals sheet and nurse-driven protocols. Catheter removal is also strongly advised if there is any suspicion of bloodstream infection, such as unexplained fever and signs of sepsis. Scheduled catheter replacement or exchange has been studied as a potential strategy to prevent CLABSIs. However, a randomized trial investigated a strategy of using scheduled catheter exchange over a guidewire or replacement at a new site after 3 days versus exchange or replacement when clinically indicated and found no difference in the rate of CRBSI[26]. This was later followed by a systematic review of scheduled replacement strategies compared to clinically indicated removal of CVCs and found no difference in CLABSI rates, although mechanical complications were higher in the groups of patients with scheduled catheter replacements[27]. To date, there are no data published in support of a scheduled replacement strategy on any schedule as an effective means for the prevention of CLABSI.

Guidewire Exchange-Catheter replacement over a guidewire is an accepted strategy for replacing malfunctioning catheters or replacing specialty catheters (such as a pulmonaryarterial catheter or a sheath introducer) with a standard central venous catheter. However, in the setting of CLABSI, replacement with a new venous puncture site is preferred to exchange over a guidewire. In the systematic review of catheter replacements, catheters exchanged over a guidewire were found to have a higher rate of colonization, exit site 
infection, and catheter-related bacteremia than those placed at a new site[27]. There are small non-randomized studies suggesting that, in difficult circumstances where obtaining access is difficult or may lead to further long-term complications (such as dialysis patients), exchange over a guidewire may be considered[28, 29].

\section{Insertion Technique}

A sterile insertion technique is absolutely crucial to maintaining low rates of CLABSI. In a large multicenter cohort study, the rate of CRBSI was followed over the course of implementation of a five171 point evidence-based strategy developed by the CDC[24]. This included the following measures (Table 2) accompanied by an aggressive education campaign targeted to clinicians and a third-party compliance observer:

By three months from the beginning of the intervention, the median rate of CRBSI had decreased from 2.7 infections per 1000 catheter-days to $0(\mathrm{p}=<0.002)$. The regression model showed a significant decrease in infection rates from baseline, with incidence-rate ratios continuously decreasing from 0.62 (95\% confidence interval [CI], 0.47 to 0.81 ) at 0 to 3 months after implementation of the intervention to 0.34 (95\% CI, 0.23 to 0.50 ) at 16 to 18 months. Overall, a sustained reduction of $66 \%$ was observed-emphasizing the importance of these basic measures in preventing CRBSI.

\section{Catheter Dressing and Maintenance}

Chlorhexidine-Impregnated Dressings-Two types of chlorhexidine gluconateimpregnated dressings are widely available: the Biopatch (http://www.ethicon.com/ healthcare-professionals/infection-prevention/biopatch-protective-disk-chg) and the Tegaderm ${ }^{\mathrm{TM}}$ CHG Chlorhexidine Gluconate IV Securement Dressing (http://www. 3m.com/3M/en_US/company-us/all-3m-products/ /3M-Tegaderm-CHG-ChlorhexidineGluconate-I-V-Securement-Dressing?N=5002385+3293321978\&rt=rud). Both are considered to be members of the same product class as they both have chlorhexidine as the active agent, both have the same anatomic site of action (skin around catheter insertion site), both have local elution of chlorhexidine directly to the catheter insertion site and surrounding skin, and both have a similar time span of delivery (e.g. while dressing is present) [30]. Both have been shown to reduce catheter colonization and catheter related infections[31-34]. A Cochrane review found moderate-quality evidence showing that chlorhexidine impregnated dressings reduce the frequency of catheter-related BSI per 1000 patient days compared with standard polyurethane dressings (RR $0.51,95 \%$ CI 0.33 to 0.78 ) and reduce catheter tip colonization compared to standard dressings (RR $0.58,95 \%$ CI 0.47 to 0.73$)[35]$.

Needleless Securement Devices-During the course of its use, CVCs commonly encounter shear forces capable of shifting catheter position or dislodging the device. Examples range from gentle forces—such as gentle tugging from manipulation-to stronger forces like accidental pulls/entanglements or pulls from agitated or delirious patients. For securement, CVCs are commonly sutured to the surrounding skin to prevent accidental dislodging. While a secure way to ensure catheters remain in place, it does increase the potential for localized skin infections and can be uncomfortable for patients. Sutureless 
securement devices (SSDs) are systems that can achieve this goal without violating the skin. SSDs secure catheters to the skin using a strong adhesive and often use the catheter hub suture wings as a key contact area [36]. Examples include the Bard StatLock CV Plus Stabilization Device (http://www.bardaccess.com/products/stabilization/other-universalplus) and the 3M PICC/CVC Needleless Securement Device (http://www.3m.com/3M/ en_US/company-us/all-3m-products/ /All-3M-Products/Health-Care/Medical/SecurementImmobilization-Dressing-Securement/Securement-Devices/? $\mathrm{N}=5002385+8707795+8707798+8710892+8711017+8711097+3294857497 \& \mathrm{rt}=\mathrm{r} 3)$. A growing body of literature has described how these devices can be used as safe and effective alternatives to suturing CVCs and other vascular access catheters, reducing catheter dislodgement, CLABSI and occupational needlestick injury[37-39]. Given the benefits of using SSDs in reducing CLABSI, they are recommended by guidelines for the securement of CVCs[1].

Disinfection Caps-Catheter manipulation for drug administration presents an opportunity for contamination, and preventative measures should be taken to prevent the potential transmission of pathogenic organisms. When access ports are manipulated, they should be scrubbed with an antiseptic solution, such as chlorhexidine, povidone iodine, an iodophor, or $70 \%$ alcohol[1]. The optimum duration of scrubbing is not known. However, a study comparing scrub times of 3, 10 and 15 seconds with $70 \%$ alcohol on catheters contaminated with a solution containing Staphylococcus aureus, Staphylococcus, Escherichia coli, and Pseudomonas aeruginosa species showed a reduction of nearly 20 fold in CFUs/mL between the 3 second time and the 15 second time[40]. This difference did not reach statistical significance $(p=0.09)$ though the study was likely under-powered. The difference in effectiveness between scrub times introduce human factors into successful decontamination efforts. In contrast, passive hub decontamination relies on hub and port covers that maintain contact between catheter hubs and disinfecting solution (http://www. 3m.com/3M/en_US/company-us/all-3m-products/ /All-3M-Products/Health-Care/Medical/ Curos/?N=5002385+8707795+8707798+8711017+8717585+3294857497\&rt=r3)

They do not require scrubbing prior to infusions or draws once they are removed. Several disinfection cap products are available and have been shown to be effective in reducing hub colonization and CLABSI. In a retrospective study investigating the introduction of disinfection cap, CLABSI rates dropped from 1.682/1000 catheter days to $0.6461 / 1000$ catheter days after implementing disinfection caps, achieving statistical significance[41]. In an observational study in an oncology unit, a total of 3,005 catheter-days and 1 CLABSI (0.3 infections/1,000 catheter-days) were documented during an intervention period where alcohol-impregnated port-protectors were introduced, compared with 6,851 catheter-days and 16 CLABSIs (2.3 infections/1,000 catheter-days) during the control period (RR, 0.14; 95\% CI, 0.02-1.07; $\mathrm{p}=.03$ )[42]. As an effective antiseptic method that does not rely on active human intervention, disinfection caps are an attractive way to complement a comprehensive CLABSI reduction strategy. 


\section{Conclusions}

Preventing CLABSIs in the ICU usually requires multiple strategies. Insertion strategies including education and training of those who insert catheters, use of chlorhexidine for skin antisepsis, and use of maximal sterile barrier precautions have a long record of preventing CLABSI. Use of novel technologies such as antibiotic or antiseptic impregnated catheters, sutureless securement devices, and disinfection caps should be added to the armamentarium of tools to further reduce CLABSI rates.

\section{References}

1. O'Grady NP, et al. Guidelines for the prevention of intravascular catheter-related infections. Clin Infect Dis. 2011; 52(9):e162-93. [PubMed: 21460264]

2. https://www.cdc.gov/nhsn/acute-care-hospital/clabsi/index.html

3. Mayer J, et al. Agreement in classifying bloodstream infections among multiple reviewers conducting surveillance. Clin Infect Dis. 2012; 55(3):364-70. [PubMed: 22539665]

4. CDC National and State Healthcare Progress Report published. Mar. 2014 accessed at https:// www.cdc.gov/HAI/pdfs/progress-report/hai-progress-report.pdf

5. Umscheid CA, et al. Estimating the proportion of healthcare-associated infections that are reasonably preventable and the related mortality and costs. Infect Control Hosp Epidemiol. 2011; 32(2):101-14. [PubMed: 21460463]

6. O'Grady NP, et al. Guidelines for the prevention of intravascular catheter-related infections. Centers for Disease Control and Prevention. MMWR Recomm Rep. 2002; 51(Rr-10):1-29.

7. CDC National and State Healthcare-Associated Infections Progress Report, published. Mar. 2014 available at http://www.cdc.gov/HAI/pdfs/progress-report/hai-progressreport.pdf

8. El-Kersh K, et al. Open and closed models of intensive care unit have different influences on infectious complications in a tertiary care center: A retrospective data analysis. Am J Infect Control. 2016

9. Parienti JJ, et al. Meta-analysis of subclavian insertion and nontunneled central venous catheterassociated infection risk reduction in critically ill adults. Crit Care Med. 2012; 40(5):1627-34. [PubMed: 22511140]

10. Parienti JJ, et al. Intravascular Complications of Central Venous Catheterization by Insertion Site. N Engl J Med. 2015; 373(13):1220-9. [PubMed: 26398070]

11. Parienti JJ, et al. Femoral vs jugular venous catheterization and risk of nosocomial events in adults requiring acute renal replacement therapy: a randomized controlled trial. Jama. 2008; 299(20): 2413-22. [PubMed: 18505951]

12. Merrer J, et al. Complications of femoral and subclavian venous catheterization in critically ill patients: a randomized controlled trial. Jama. 2001; 286(6):700-7. [PubMed: 11495620]

13. Rabindranath KS, et al. Use of real-time ultrasound guidance for the placement of hemodialysis catheters: a systematic review and meta-analysis of randomized controlled trials. Am J Kidney Dis. 2011; 58(6):964-70. [PubMed: 22099570]

14. Brass $P$, et al. Ultrasound guidance versus anatomical landmarks for subclavian or femoral vein catheterization. Cochrane Database Syst Rev. 2015; 1:Cd011447. [PubMed: 25575245]

15. Early TF, et al. Increased infection rate in double-lumen versus single-lumen Hickman catheters in cancer patients. South Med J. 1990; 83(1):34-6. [PubMed: 2300831]

16. Yeung C, May J, Hughes R. Infection rate for single lumen v triple lumen subclavian catheters. Infect Control Hosp Epidemiol. 1988; 9(4):154-8. [PubMed: 3129491]

17. Templeton A, et al. Multilumen central venous catheters increase risk for catheter-related bloodstream infection: prospective surveillance study. Infection. 2008; 36(4):322-7. [PubMed: 18663408]

18. Dezfulian C, et al. Rates of infection for single-lumen versus multilumen central venous catheters: a meta-analysis. Crit Care Med. 2003; 31(9):2385-90. [PubMed: 14501971] 
19. Harron K, et al. Generalisability and Cost-Impact of Antibiotic-Impregnated Central Venous Catheters for Reducing Risk of Bloodstream Infection in Paediatric Intensive Care Units in England. PLoS One. 2016; 11(3):e0151348. [PubMed: 26999045]

20. Gilbert RE, et al. Impregnated central venous catheters for prevention of bloodstream infection in children (the CATCH trial): a randomised controlled trial. Lancet. 2016; 387(10029):1732-42. [PubMed: 26946925]

21. Veenstra DL, Saint S, Sullivan SD. Cost-effectiveness of antiseptic-impregnated central venous catheters for the prevention of catheter-related bloodstream infection. Jama. 1999; 282(6):554-60. [PubMed: 10450717]

22. Brun-Buisson C, et al. Prevention of intravascular catheter-related infection with newer chlorhexidine-silver sulfadiazine-coated catheters: a randomized controlled trial. Intensive Care Med. 2004; 30(5):837-43. [PubMed: 15060765]

23. Lai NM, et al. Catheter impregnation, coating or bonding for reducing central venous catheterrelated infections in adults. Cochrane Database Syst Rev. 2016; 3:Cd007878. [PubMed: 26982376]

24. Pronovost $\mathrm{P}$, et al. An intervention to decrease catheter-related bloodstream infections in the ICU. N Engl J Med. 2006; 355(26):2725-32. [PubMed: 17192537]

25. Berenholtz SM, et al. Eliminating catheter-related bloodstream infections in the intensive care unit. Crit Care Med. 2004; 32(10):2014-20. [PubMed: 15483409]

26. Cobb DK, et al. A controlled trial of scheduled replacement of central venous and pulmonaryartery catheters. N Engl J Med. 1992; 327(15):1062-8. [PubMed: 1522842]

27. Cook D, et al. Central venous catheter replacement strategies: a systematic review of the literature. Crit Care Med. 1997; 25(8):1417-24. [PubMed: 9267959]

28. Chaftari AM, et al. The use of minocycline-rifampin coated central venous catheters for exchange of catheters in the setting of staphylococcus aureus central line associated bloodstream infections. BMC Infect Dis. 2014; 14:518. [PubMed: 25253042]

29. Hou SM, et al. Is guidewire exchange a better approach for subclavian vein re-catheterization for chronic hemodialysis patients? Thromb Res. 2006; 118(4):439-45. [PubMed: 16214203]

30. [accessed March21,2017] U.S. Department of Health and Human Services Centers for Disease Control and Prevention HIPAC Meeting. Nov. 2015 http://www.cdc.gov/HICPAC/ presentations.html

31. Arvaniti K, et al. Comparison of Oligon catheters and chlorhexidine-impregnated sponges with standard multilumen central venous catheters for prevention of associated colonization and infections in intensive care unit patients: a multicenter, randomized, controlled study. Crit Care Med. 2012; 40(2):420-9. [PubMed: 21926583]

32. Ruschulte $\mathrm{H}$, et al. Prevention of central venous catheter related infections with chlorhexidine gluconate impregnated wound dressings: a randomized controlled trial. Ann Hematol. 2009; 88(3): 267-72. [PubMed: 18679683]

33. Timsit JF, et al. Randomized controlled trial of chlorhexidine dressing and highly adhesive dressing for preventing catheter-related infections in critically ill adults. Am J Respir Crit Care Med. 2012; 186(12):1272-8. [PubMed: 23043083]

34. Timsit JF, et al. Chlorhexidine-impregnated sponges and less frequent dressing changes for prevention of catheter-related infections in critically ill adults: a randomized controlled trial. Jama. 2009; 301(12):1231-41. [PubMed: 19318651]

35. Ullman AJ, et al. Dressings and securement devices for central venous catheters (CVC). Cochrane Database Syst Rev. 2015; (9):Cd010367. [PubMed: 26358142]

36. Krenik KM, Smith GE, Bernatchez SF. Catheter Securement Systems for Peripherally Inserted and Nontunneled Central Vascular Access Devices: Clinical Evaluation of a Novel Sutureless Device. J Infus Nurs. 2016; 39(4):210-7. [PubMed: 27379679]

37. Stephenson C. The Advantages of a Precision-engineered Securement Device for Fixation of Arterial Pressure-monitoring Catheters. The Journal of the Association for Vascular Access. 10(3): $130-132$.

38. Frey AM, Schears GJ. Why are we stuck on tape and suture? A review of catheter securement devices. J Infus Nurs. 2006; 29(1):34-8. [PubMed: 16428999] 
39. Yamamoto AJ, et al. Sutureless securement device reduces complications of peripherally inserted central venous catheters. J Vasc Interv Radiol. 2002; 13(1):77-81. [PubMed: 11788698]

40. Simmons, S., Bryson, C., Porter, S. Crit Care Nurs Q. United States: 2011. "Scrub the hub": cleaning duration and reduction in bacterial load on central venous catheters; p. 31-5.

41. Schears G. "Cap the connector: save the patient," in Proceedings of the AVA Annual Scientific Meeting. 2011

42. Sweet MA, et al. Impact of alcohol-impregnated port protectors and needleless neutral pressure connectors on central line-associated bloodstream infections and contamination of blood cultures in an inpatient oncology unit. Am J Infect Control. 2012; 40(10):931-4. [PubMed: 22575286] 


\section{Key Points}

- $\quad$ The incidence of CLABSI has decreased with the implementation of evidence-based practice guidelines.

- Clinical factors that may reduce the risk of CLABSI include catheter choice, catheter site selection, insertion technique, and catheter maintenance.

- $\quad$ Newer technology, such as needleless securement devices and disinfecting caps have been shown to be additional effective strategies to further reduce the incidence of CLABSI. 


\section{Table 1}

\section{Comparison of CRBSI and CLABSI}

\begin{tabular}{|l|l|}
\hline CRBSI & $\begin{array}{l}\text { Clinical signs of sepsis and positive peripheral blood culture in absence of an obvious source other than CVC with one of the } \\
\text { following: }\end{array}$ \\
$\begin{array}{r}\text { Positive semi-quantitative }(>15 \mathrm{CFU}) \text { or quantitative }\left(>10^{3} \mathrm{CFU}\right) \text { culture from a catheter segment with the same } \\
\text { organisms isolated peripherally } \\
\text { Simultaneous quantitative blood cultures with a ratio of } 23: 1 \text { (CVC vs. peripheral) } \\
\text { Time to culture positivity difference no more than } 2 \mathrm{~h} \text { between } \mathrm{CVC} \text { cultures and peripheral cultures }\end{array}$ \\
\hline CLABSI & $\begin{array}{l}\text { Primary bloodstream infection in a patient that had a central line within the } 48 \text {-hour period before development } \\
\text { Infection must not be related to an alternative cause }\end{array}$ \\
\hline
\end{tabular}




\section{Table 2}

\section{Evidence-Based Strategies to Reduce CLABSI}

\begin{tabular}{|ll|}
\hline $\mathbf{1}$ & Handwashing with soap and water \\
$\mathbf{2}$ & Sterile insertion with full barrier precautions (cap, mask, sterile gown, sterile gloves and full sterile drape) \\
$\mathbf{3}$ & Use of $2 \%$ chlorhexidine solution with proper air drying before insertion \\
$\mathbf{4}$ & Avoiding femoral site for catheterization \\
$\mathbf{5}$ & Prompt removal of unnecessary catheters
\end{tabular}

